\title{
Efficacy of interventions for amblyopia: a systematic review and network meta- analysis
}

Yonghong $\mathrm{Li}^{1 \dagger}$, Huan Sun ${ }^{2 \dagger}$, Xiaojuan $\mathrm{Zhu}^{3}$, Yana Su${ }^{1}$, Tianqi Yu', Xinyu $\mathrm{Wu}^{1}$, Xiaoqin Zhou ${ }^{4}$ and $\mathrm{Li} \mathrm{Jing}^{1 *}$ (D)

\begin{abstract}
Background: Many treatments are currently available for amblyopic patients; although, the comparative efficacy of these therapies is unclear. We conducted a systematic review and network meta-analysis (NMA) to establish the relative efficacy of these treatments for amblyopia.

Methods: Electronic databases (MEDLINE, EMBASE, Cochrane Library) were systematically searched from inception to Sep. 2019. Only Randomized clinical trials comparing any two or three of the following treatments were included: refractive correction (spectacles alone), patching of $2 \mathrm{~h}$ per day (patch $2 \mathrm{H}$ ), patch $6 \mathrm{H}$, patch $12 \mathrm{H}$, patch $2 \mathrm{H}+$ near activities $(\mathrm{N})$, patch $2 \mathrm{H}+$ distant activities $(\mathrm{D})$, atropine (Atr) daily, Atr weekly, Atr weekly + plano lens over the sound eye (Plano), optical penalization and binocular therapy. The reviewers independently extracted the data according to the PRISMA guidelines; assessed study quality by Cochrane risk-of-bias tool for randomized trials. The primary outcome measure was the change in best-corrected visual acuity (BCVA) expressed as log MAR lines. Direct comparisons and a Bayesian meta-analysis were performed to synthesize data.
\end{abstract}

Results: Twenty-three studies with 3279 patients were included. In the network meta-analysis, optical penalization was the least effective of all the treatments for the change of visual acuity, spectacles (mean difference [MD], 2.9 Log MAR lines; 95\% credibility interval [Crl], 1.8-4.0), patch 2H (MD, 3.3; 95\% Crl, 2.3-4.3), patch 6H (MD, 3.6; 95\% Crl, 2.6-4.6), patch $12 \mathrm{H}(\mathrm{MD}, 3.4 ; 95 \% \mathrm{Crl}, 2.3-4.5)$, patch $2 \mathrm{H}+\mathrm{N}(\mathrm{MD}, 3.7 ; 95 \% \mathrm{Crl}, 2.5-5.0)$, patch $2 \mathrm{H}+\mathrm{D}(\mathrm{MD}, 3.5 ; 95 \%$ $\mathrm{Crl}, 2.1-5.0$ ), Atr daily (MD, 3.2; 95\% Crl, 2.2-4.3), Atr weekly (MD, 3.2; 95\% Crl, 2.2-4.3), Atr weekly + Plano (MD, 3.7; $95 \% \mathrm{Crl}, 2.7-4.7)$, binocular therapy $(\mathrm{MD}, 3.1 ; 95 \% \mathrm{Crl}, 2.0-4.2)$. The patch $6 \mathrm{H}$ and patch $2 \mathrm{H}+\mathrm{N}$ were better than spectacles ([MD, 0.73; 95\% Crl, 0.10-1.40]; [MD, 0.84; 95\% Crl, 0.19-1.50]).

Conclusions: The NMA indicated that the efficacy of the most of the examined treatment modalities for amblyopia were comparable, with no significant difference. Further high quality randomized controlled trials are required to determine their efficacy and acceptability.

Systematic review registration: CRD42019119843.

Keywords: Amblyopia, Spectacles, Patching, Atropine, Optical penalization, Binocular, Meta-analysis

\footnotetext{
* Correspondence: lijing68@hotmail.com

${ }^{\dagger}$ Yonghong Li and Huan Sun contributed equally to this work.

'Department of Evidence-Based Medicine and Clinical Epidemiology, West

China Hospital, Sichuan University, No.37 Guoxue Alley, Chengdu 610041,

China

Full list of author information is available at the end of the article
}

(c) The Author(s). 2020 Open Access This article is licensed under a Creative Commons Attribution 4.0 International License, which permits use, sharing, adaptation, distribution and reproduction in any medium or format, as long as you give appropriate credit to the original author(s) and the source, provide a link to the Creative Commons licence, and indicate if changes were made. The images or other third party material in this article are included in the article's Creative Commons licence, unless indicated otherwise in a credit line to the material. If material is not included in the article's Creative Commons licence and your intended use is not permitted by statutory regulation or exceeds the permitted use, you will need to obtain permission directly from the copyright holder. To view a copy of this licence, visit http://creativecommons.org/licenses/by/4.0/ The Creative Commons Public Domain Dedication waiver (http://creativecommons.org/publicdomain/zero/1.0/) applies to the data made available in this article, unless otherwise stated in a credit line to the data. 


\section{Background}

Amblyopia is a type of neurodevelopmental disorder that constitute a largest threat to the vision of children. The prevalence of amblyopia is estimated at $1-5 \%$ in different areas and studies [1-3]. And amblyopia can lead to permanently reduced vision if not treated properly. Based on the current evidence, amblyopia is mainly caused by uncorrected refractive error, strabismus, cataract and ptosis [4]. There is a plethora of visual function deficits of the amblyopic individual that could affect learning, daily activities and psychological state of affected children $[5,6]$. Therefore, it is essential to get the best treatment during the critical period to avoid severe consequences later on due to this disorder.

More than ten therapeutic regimens have been used to treat amblyopia in clinics based on the theory of visual stimulation [4, 7-11]. Among them, conventional patching is still mainly used in clinical settings for its long-term, relative safety. However, the patching regimen usually depends on the ophthalmologists' clinical impressions, training, and observations [7]. In addition, recent studies found that most of the adverse implications are caused by the treatment rather than the condition itself; patching is more likely to impact psycho-social and quality of life both for the child and the family $[5,12]$. Innovative methods including pharmacological penalization and the binocular approach using virtual reality software and devices are not widely accepted and used currently, even though some studies have shown that these treatments are not less effective than patching [7]. To achieve better outcomes in both vision improvement and in reduction of adverse reactions, it is important to determine more clearly the effects of these different treatment paradigms.

Not all treatments have been directly compared and many randomized controlled trials (RCTs) and metaanalyses showed no difference between the specific treatments compared [13-18]. Therefore, we provide an NMA that allows for both direct and indirect comparisons to further clarify the efficacy of current interventions for amblyopia.

\section{Methods}

A study protocol was registered with PROSPERO (CRD42019119843). This review adheres to the PRISMA extension statement for network meta-analyses [19].

\section{Data sources and search strategy}

The Ovid MEDLINE, Ovid EMBASE, Cochrane Central Register of Controlled Trials (CENTRAL) were systematically searched to include relevant studies published in English from inception to 1st Sep. 2019 (see Additional file 1). The Cochrane Database of Systematic Reviews and reference lists of published reviews were screened to identify additional relevant studies. The searches were independently performed by the researchers and disagreements were discussed and resolved by consensus.

\section{Eligibility criteria}

Included were the RCTs that enrolled patients with strabismus, anisometropia, mixed and residual amblyopia. Trials that compared at least two of the following 11 interventions were eligible: refractive correction (spectacles alone), patch $2 \mathrm{~h}$ per day $(2 \mathrm{H})$, patch $6 \mathrm{H}$, patch $12 \mathrm{H}$, patch $2 \mathrm{H}+$ near activities $(\mathrm{N})$, patch $2 \mathrm{H}+$ distant activities (D), atropine (Atr) daily, Atr weekly, Atr weekly + plano lens over the sound eye (Plano), optical penalization and binocular therapy. All participants wore spectacles if prescribed (the detailed therapeutic regimens were shown in Additional file 2).

Exclusion criteria were study designs different from this study, interventions different from this study such as Bangerter filters or Amblyz liquid crystal occlusion glasses, no specific intervention time and data unsuitable for meta-analysis.

\section{Data collection and outcome measures}

Researchers independently screened articles and extracted the data according to the inclusion criteria and the data extraction form used. When the same population were involved in multiple study publications, only the primary report was included in the meta-analysis. The outcome measure was the improvement of best-corrected visual acuity (BCVA) in the amblyopic eye expressed as log MAR units. The BCVA was obtained by a study-certified examiner using the Early Treatment Diabetic Retinopathy Study (ETDRS) protocol or Amblyopia Treatment Study VA-testing (ATS-HOTV) protocol [20, 21].

The following data were extracted: first author, year of publication, baseline demographic characteristics (age, sex, visual acuity and types of amblyopia), interventions, sample sizes, duration of treatment as well as outcomes. In addition, the characteristics of the study design were extracted to assess the risk of bias within included studies.

\section{Risk of Bias assessment}

The risk of bias of included studies was assessed independently by the authors according to the seven domains of Cochrane Risk of Bias Tool for randomized trials [22]: random sequence generation, allocation concealment, blinding of the participants and personnel, blinding of outcome assessment, incomplete outcome data, selective outcome reporting and other biases.

\section{Data synthesis and analysis}

Pairwise meta-analysis for all direct comparisons were performed using the random-effects model by Review Manager 5.3. Thereafter, the network meta-analysis was conducted based on a Bayesian framework random-effects model. The process of model specification, priors setting, starting values selection of multiple chains were automatically completed through Gemtc package [23], by which 
the consistency model was selected. Markov Chain Monte Carlo (MCMC) simulation was computed by running four chains simultaneously with 10,000 iterations discarded and 40,000 iterations obtained finally to achieve stability. The model convergence was checked by Brooks-GelmanRubin diagnostic statistics with potential scale reduction factor (PSRF) less than 1.05 considered acceptable, and through inspection of trace plot and density plots [24].

The validity of indirect and mixed comparisons should consider three main assumptions: heterogeneity, inconsistency and transitivity. The global heterogeneity was assessed using Chi-square test and $I^{2}$ statistic, with $I^{2}$ values greater than $75 \%$ indicating substantial heterogeneity, and the heterogeneity factor $\left(\tau^{2}\right)$ was also calculated. The researchers planned to assess the inconsistency for each comparison by the node-splitting approach with two-side $p$-values $<0.05$ considered statistically significant [25]. For the transitivity, only the clinical and methodological comparability of included studies can be described while there are no statistical methods for testing.

The results of continuous outcomes were expressed as mean differences (MD) with $95 \%$ credibility intervals $(\mathrm{CrI})$, and the outcomes were interpreted as significant when the $95 \% \mathrm{Crl}$ excludes the null value. The network meta-analysis was conducted in $\mathrm{R}$ software (version 3.4.0) interfacing with JAGS (version 4.3.0) [23]. The ranking probabilities of all interventions were estimated and the surface under the cumulative ranking curve (SUCRA) was provided with Stata (version 13.0) [26] (analysis code is placed in Additional file 3).

Researchers planned three sensitivity analyses for the change of amblyopic BCVA by removing (1) highly heterogeneous studies that lead heterogeneity greater than $75 \%$, (2) studies with residual amblyopia and (3) studies with patients beyond 13 years of age.

\section{Results}

\section{Characteristics and risk of bias of the included studies}

The PPSISMA diagram for systematic search and screening is shown in Fig. 1. Of 1629 relevant records, twenty-three studies with a total of 3279 patients were included in this network meta-analysis [27-49]. One (4.3\%) of the studies contains two independent trials, one study was three-arm trial, and thirteen studies (56.5\%) came from the Pediatric Eye Disease Investigator Group [50]. There were 15 comparisons among 11 different treatments (Fig. 2). Seventeen (70.8\%) trials included patients under 10 years of age. All types of

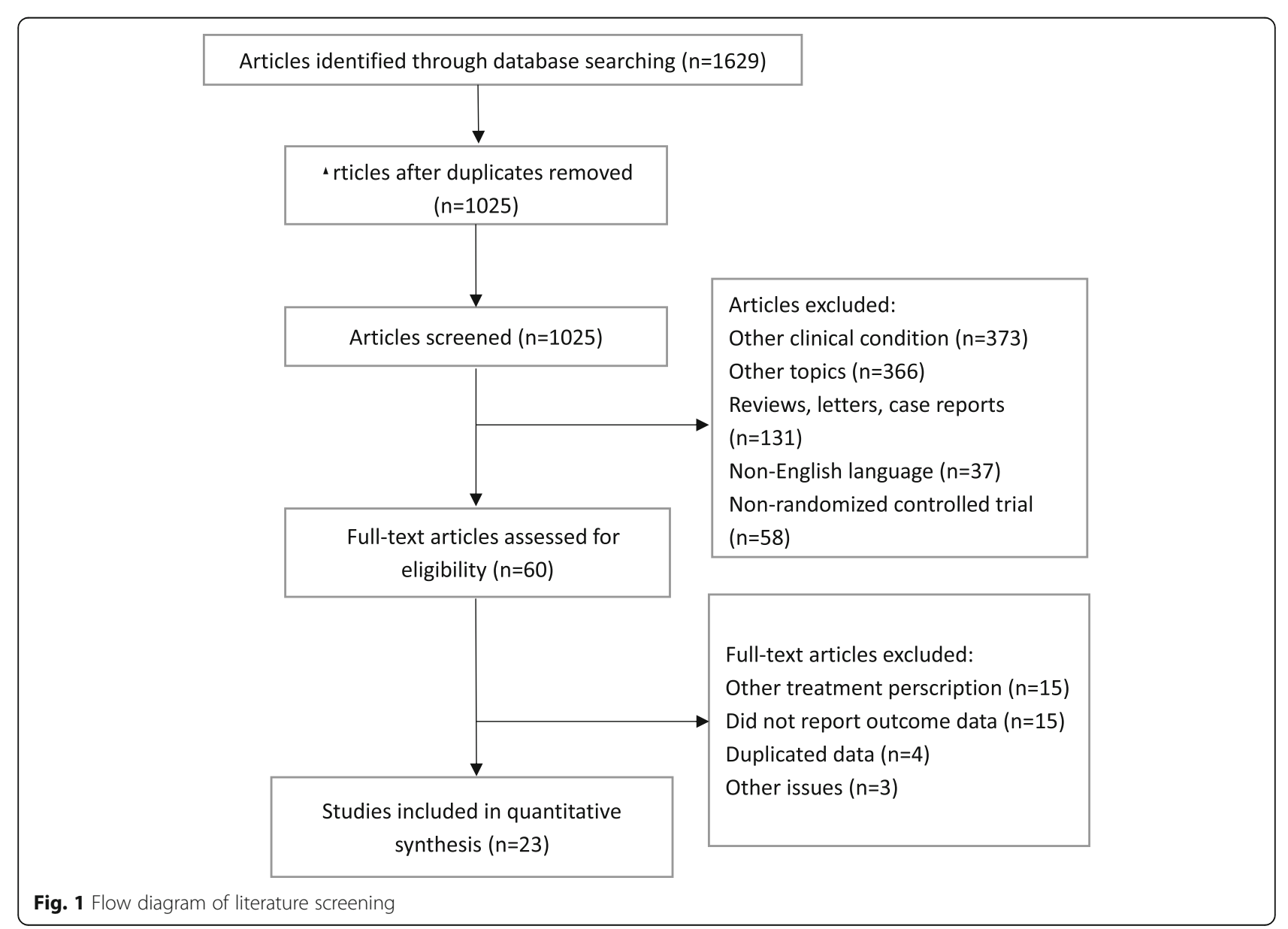




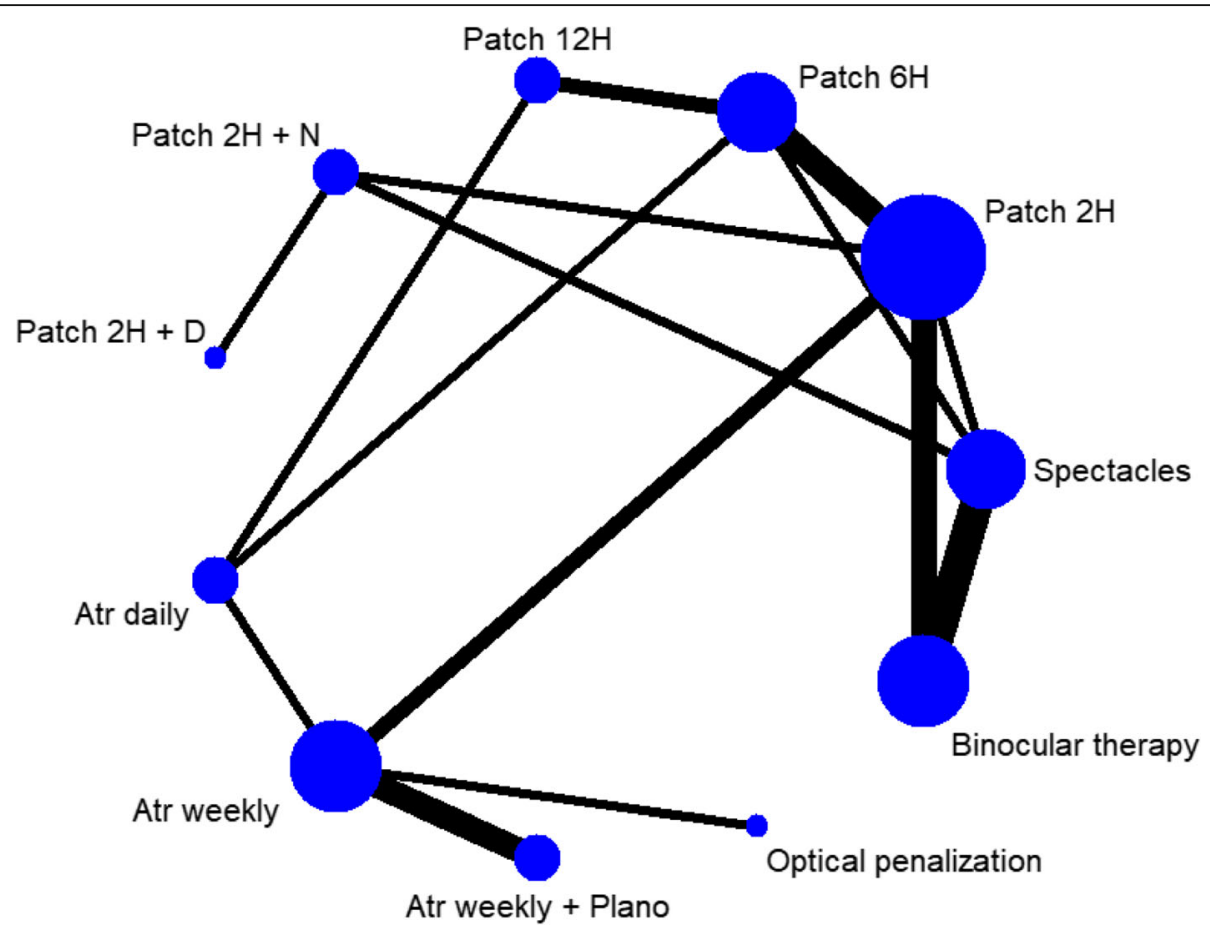

Fig. 2 Network of eligible comparisons. The size of each circle represents the number of studies for the treatment, the line thickness of each connection denotes the number of studies investigating the comparison. $\mathrm{H}$, hours per day; Atr, atropine; $\mathrm{N}$, near activities; $\mathrm{D}$, distant activities; Plano, plano lens over the sound eye

amblyopia were studied in seventeen $(73.9 \%)$ studies, while one $(4.3 \%)$ studies only included anisometropia, one (4.3\%) study only included strabismus and two (8.6\%) studies focused on residual amblyopia. The average follow-up time was 12.9 weeks (see Additional file 4).

We found low to moderate risk of bias in included studies, with $20(86.9 \%)$ of studies reported adequate random sequence generation, 15 (65.2\%) blinded outcome assessment and 20 (86.9\%) reported all the randomized participants outcomes. However, most of the studies $(86.9 \%)$ did not mention the allocation concealment; uncertainty about the reporting bias does exist because we had insufficient imformation to assess the risk of selective reporting bias in nine studies. In addition, some of the participants in studies had received prior treatments before the trial, which might increase the risk of selection bias (see Additional file 5).

\section{Direct meta-analysis}

In direct comparisons, we only found that atropine weekly was associated with a better effect on the change of BCVA compared with optical penalization (MD, $3.22 \log$ MAR lines; 95\% CI, 2.72-3.72); the atropine weekly combined with a plano lens was more noticeable in improving visual acuity than that with atropine weekly alone (MD 0.44; 95\% CI, 0.05-0.83) (Table 1).

\section{Network meta-analysis}

In the network comparisons of the improvement of BCVA, the optical penalization was inferior to spectacles (MD, 2.90; 95\% CrI, 1.80-4.00), patch 2H (MD, 3.30; 95\% CrI, 2.30-4.30), patch 6H (MD, 3.60; 95\% CrI, 2.60-4.60), patch $12 \mathrm{H}$ (MD, 3.40; 95\% CrI, 2.30-4.50), patch $2 \mathrm{H}+\mathrm{N}(\mathrm{MD}, 3.70 ; 95 \% \mathrm{CrI}, 2.50-5.00)$, patch $2 \mathrm{H}+\mathrm{D}$ (MD, 3.50; 95\% CrI, 2.10-5.00), Atr daily (MD, 3.20; 95\% CrI, 2.20-4.30), Atr weekly (MD, 3.20; 95\% CrI, 2.40-4.00), Atr weekly + Plano (MD, 3.70; 95\% CrI, $2.70-4.70)$ and binocular therapy (MD, 3.10; 95\% CrI, 2.00-4.20). When compared with spectacles, only patch $6 \mathrm{H}$, and patch $2 \mathrm{H}+\mathrm{N}$ showed better effectiveness with a MD of 0.73 (95\% Crl, 0.10-1.40) and 0.84 (95\% CrI, 0.19-1.50), respectively. No other statistical difference was found (Fig. 3). According to the rank probability and SUCRAs, patch $2 \mathrm{H}+\mathrm{N}$ had the highest probability to be the best treatment which would result in greater improvements of BCVA. Thereafter, Atr weekly + Plano, patch $6 \mathrm{H}$, and patch $2 \mathrm{H}+\mathrm{D}$ were ranked in the next three positions (see Additional file 6).

There was no considerable heterogeneity in the direct metaanalysis except the comparison of spectacles versus binocular treatment $\left(I^{2}=95.57 \%\right)$. The global $I^{2}$ was estimated to be zero. It was found that the direct and indirect results were consistent for the change of BCVA by using node-splitting approach (range of $p$ values: $0.22-0.84$ ) (see Additional file 7). 
Table 1 The results of direct meta-analysis

\begin{tabular}{llll}
\hline Comparisons & No. of Trials & No. of Patients & Mean Difference ${ }^{\mathbf{a}}(95 \%$ Cl) \\
\hline Spectacles vs Patch $2 \mathrm{H}$ & 1 & 35 & $-0.51(-1.70,0.71)$ \\
Spectacles vs Patch 6H & 1 & 35 & $-1.00(-2.40,0.38)$ \\
Spectacles vs Patch 2H + N & 1 & 173 & $-0.60(-1.40,0.21)$ \\
Patch 2H vs Patch 2H + N & 1 & 64 & $-1.00(-2.20,0.16)$ \\
Patch 6H vs Patch 12H & 1 & 157 & $0.16(-0.53,0.85)$ \\
Patch 6H vs Atr daily & 1 & 402 & $0.32(-0.43,1.10)$ \\
Patch 12H vs Atr daily & 1 & 57 & $0.05(-0.87,0.93)$ \\
Patch 2H + N vs Patch 2H + D & 1 & 392 & $0.20(-0.14,0.54)$ \\
Atr daily vs Atr weekly & 1 & 168 & $-0.20(-1.00,0.61)$ \\
Atr weekly vs Optical penalization & 1 & 63 & $\mathbf{3 . 2 2}(\mathbf{2 . 7 2 , 3 . 7 2 )}$ \\
Spectacles vs Binocular therapy & 4 & 341 & $-0.21(-0.61,0.13)$ \\
Patch 2H vs Patch 6H & 3 & 379 & $-0.38(-0.91,0.14)$ \\
Patch 2H vs Atr weekly & 2 & 205 & $0.21(-0.48,0.89)$ \\
Patch 2H vs Binocular therapy & 3 & 486 & $0.28(-0.19,0.75)$ \\
Atr weekly vs Atr weekly + Plano & 2 & 300 & $-\mathbf{0 . 4 4}(-\mathbf{0 . 8 3 , - 0 . 0 5 )}$
\end{tabular}

$H$ hours per day, Atr atropine, $N$ near activities, $D$ distant activities, Plano plano lens over the sound eye. ${ }^{a}$ For the improvement of amblyopic $B C V A, M D>1$ favored the treatment on the left side

\section{Sensitivity analyses and subgroup analysis}

In sensitivity analyses, we use spectacles as the reference intervention. After the removal of highly heterogeneous studies [43], studies including only residual amblyopia $[35,42]$ and those with patients over 13 years of age $[40,41]$, the effect value and conclusion changed slightly, whereas the ranking results of treatments in this NMA did not (Table 2). Due to the small number of studies, no subgroup analysis was carried out.

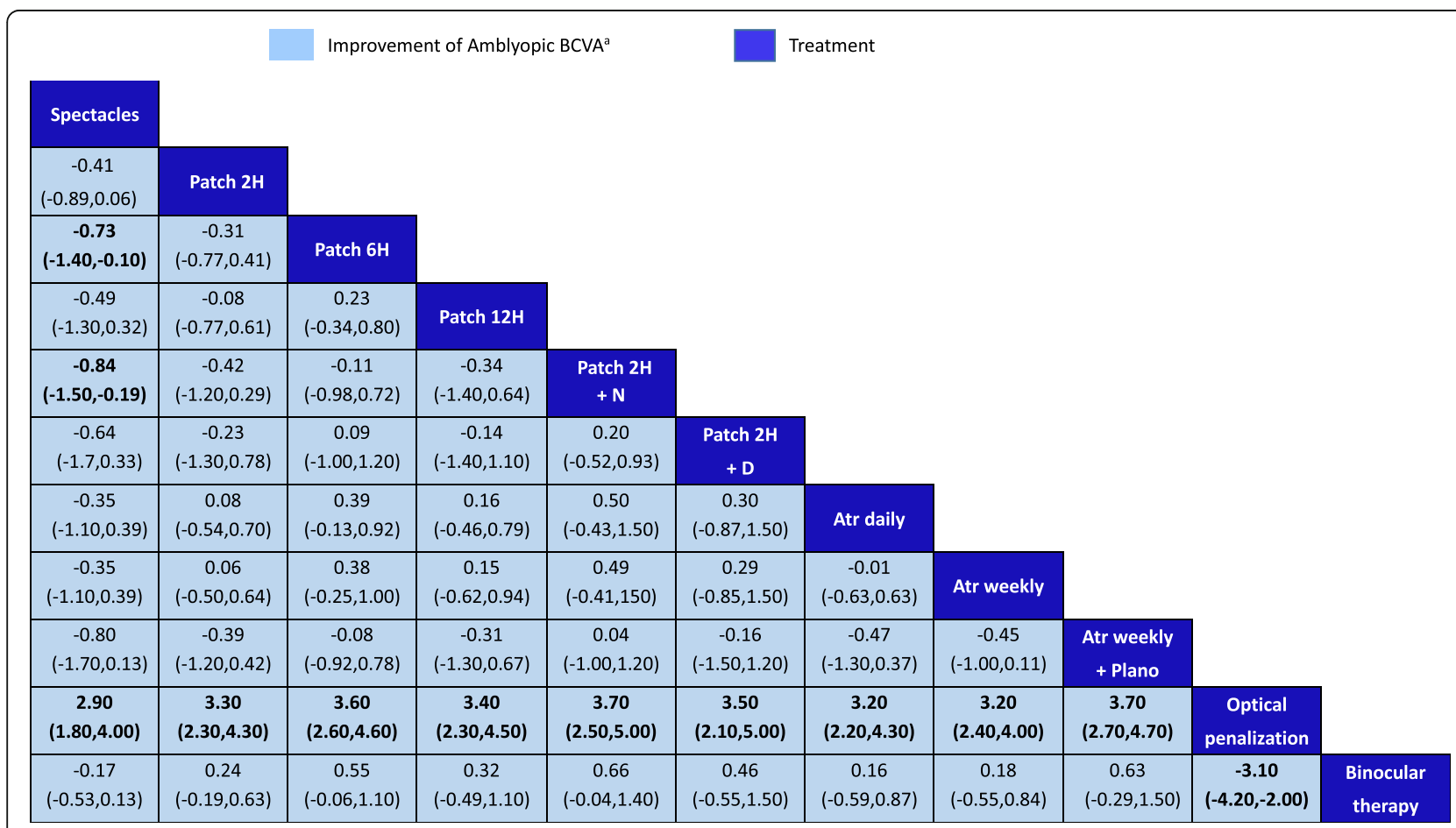

Fig. 3 Results of network meta-analysis. ${ }^{a}$ For the improvement of amblyopic BCVA, MD > 1 favored the treatments on the left side of the Table. $\mathrm{H}$, hours per day; Atr, atropine; N, near activities; D, distant activities; Plano, plano lens over the sound eye 
Table $\mathbf{2}$ The results of sensitivity analyses ${ }^{\mathrm{a}}$

\begin{tabular}{|c|c|c|c|c|}
\hline Treatment & Main analysis & $\begin{array}{l}\text { Removal of high heterogeneity } \\
\text { studies }\end{array}$ & $\begin{array}{l}\text { Removal of residual } \\
\text { amblyopia }\end{array}$ & $\begin{array}{l}\text { Removal of patients over } 13 \\
\text { years }\end{array}$ \\
\hline Patch $2 \mathrm{H}$ & $0.41[-0.06,0.89]$ & $0.32[0.02,0.56]$ & $0.42[-0.03,0.90]$ & $0.29[-0.41,0.92]$ \\
\hline Patch 6H & $0.73[0.10,1.40]$ & $0.64[0.23,1.10]$ & $0.50[-0.14,1.10]$ & $0.57[-0.35,1.40]$ \\
\hline Patch $12 \mathrm{H}$ & $0.49[-0.32,1.30]$ & $0.41[-0.17,0.96]$ & $0.29[-0.53,1.20]$ & $0.34[-0.74,1.30]$ \\
\hline Patch $2 \mathrm{H}+\mathrm{N}$ & $0.84[0.19,1.50]$ & $0.75[0.27,1.20]$ & $0.83[0.20,1.50]$ & $0.82[0.08,1.60]$ \\
\hline Patch $2 \mathrm{H}+\mathrm{D}$ & $0.64[-0.33,1.70]$ & $0.56[-0.08,1.20]$ & $0.63[-0.29,1.60]$ & $0.62[-0.49,1.70]$ \\
\hline Atr daily & $0.34[-0.43,1.10]$ & $0.25[-0.25,0.71]$ & $0.17[-0.58,0.98]$ & $0.18[-0.84,1.10]$ \\
\hline Atr weekly & $0.35[-0.39,1.10]$ & $0.27[-0.32,0.73]$ & $0.28[-0.43,1.00]$ & $0.20[-0.76,1.10]$ \\
\hline Atr weekly + Plano & $0.80[-0.13,1.70]$ & $0.72[-0.05,1.30]$ & $0.70[-0.29,1.70]$ & $0.67[-0.48,1.70]$ \\
\hline $\begin{array}{l}\text { Optical } \\
\text { penalization }\end{array}$ & $\begin{array}{l}-2.90[-4.00,- \\
1.80]\end{array}$ & $-3.00[-3.70,-2.20]$ & $-2.90[-4.00,-1.90]$ & $-3.00[-4.30,-1.80]$ \\
\hline Binocular therapy & $0.17[-0.13,0.53]$ & $0.03[-0.17,0.20]$ & $0.17[-1.20,0.53]$ & $0.18[-0.20,0.59]$ \\
\hline
\end{tabular}

$H$ hours per day, Atr atropine, $N$ near activities, $D$ distant activities, Plano plano lens over the sound eye

${ }^{a}$ Data are mean differences (MD) and $95 \% \mathrm{Crl}$ compared with refractive correction

\section{Discussions}

Although many RCTs, pairwise meta-analysis and descriptive reviews have compared the therapeutic regimens for amblyopia [4,51], it was not possible to have all regimens being compared head-to-head in a study owing to the large range of treatments. Current guidelines for the treatment of amblyopia are mostly based on the results of randomized controlled trials, since there was lack of high level evidence. Therefore, the researchers adopted the network meta-analysis techniques combined with latest RCTs to provide more guidance for the treatment of amblyopia.

Using data from twenty-four RCTs with 3279 participants, the results of direct comparisons showed that Atr weekly was worse than Atr weekly combined with plano lens over the sound eye, but better than optical penalization for the improvement of visual acuity, with a difference of 0.44 and $3.22 \log$ MAR lines, respectively. According to network meta-analysis, all the studied interventions were found to be more effective than optical penalization. In addition, patch $6 \mathrm{H}$, patch $2 \mathrm{H}+\mathrm{N}$ were more effective than refractive correction with spectacles. The ranking of efficacy is as follows: patch $2 \mathrm{H}+\mathrm{N}$, Atr weekly + Plano, patch $6 \mathrm{H}$, patch $2 \mathrm{H}+\mathrm{D}$, patch $12 \mathrm{H}$, patch $2 \mathrm{H}$, Atr weekly, Atr daily, binocular therapy, spectacles and optical penalization.

From historical research, three systematic reviews have been conducted to compare the conventional patching therapy with atropine penalization; both of the results suggested that atropine was as effective as patching in improving visual acuity $[15,52,53]$. In a head-to-head meta-analysis, there was no difference between part-time and full-time occlusion [14]. Moreover, Shotton (2008) concluded that spectacles alone is beneficial for unilateral amblyopia [54]. Taylor (2011) reported that patching appears to be more effective than spectacles while the benefit of adding near activities to patching is unproven [55]. However, it was found that most of the results were based on the descriptions of randomized controlled trials with no pooled analysis. Different from other studies is that we separated the interventions in greater detail and provided a global comparison as well as ranks of these treatments based on SUCRA values.

According to the ranking, patching regimens seem to be better than atropine except for the atropine weekly combined with a plano lens over the sound eye. Since the addition of plano lens is analogous to optical penalization or even patching, it is suggested that more studies are needed to determine the real difference between patching and atropine therapy. Consistent with previous studies [14], it was found that there was no significant difference between $2 \mathrm{~h}, 6 \mathrm{~h}$, and $12 \mathrm{~h}$ of patching, while the addition of $1 \mathrm{~h}$ of activities (near or distant) to patching seemed to be more effective than patching alone. However, activities are usually used in combination with patching and there are no RCTs to evaluate the effect of activity treatment alone.

Binocular therapy is considered to be the most important development in the field of amblyopia for the past decade $[8,56]$. To date, binocular therapy has only been compared with $2 \mathrm{~h}$ of patching and spectacles, and the present NMA result also showed that the efficacy of binocular treatment is not encouraging in improving visual acuity. Hence, using the binocular approach as a routine treatment for amblyopia is currently not recommended.

Another common studied treatment for the amblyopia is levodopa. However, this intervention was not included in this NMA due to the current levodopa treatment including a wide range of dose and duration [57]. Traditional Chinese acupuncture (TCA) could be a potential treatment for amblyopia by regulating neurotransmitters and neurotrophic factor in the visual system, and 
promoting the expression of genes related to visual plasticity [58]. Since there are few studies on acupuncture treatment and only anisometropia amblyopia was included in these studies, acupuncture was excluded from this study.

This review has several limitations. Firstly, the number of studies included are not enough to make a reliable estimate for the outcomes, several interventions had no direct comparisons and two interventions (patch $2 \mathrm{H}+\mathrm{D}$ and optical penalization) were only studied in one trial. Secondly, there was a wider range in participants ages (3 to 20 years). Some studies reported only the age range or mean, thus we were unable to perform a subgroup analysis to determine the impact of age. Likewise, the data was insufficient to analyze the effects of amblyopia severity on treatment outcome. Thirdly, all the analyses are based on the treatment duration specified in trial design or estimated rather than the actual time of patching. Moreover, other efficacy outcomes and safety of interventions were not presented in this NMA due to the lack of such information and data in the studies concerned.

Despite the valuable data gathered on this subject, several aspects remain to be considered in future researches. First and foremost, in interpreting the results of any studies and designing new studies for amblyopia treatments, there needs to be an awareness of the potential biases including age, baseline refractive error, subtype and severity of amblyopia, previous treatment as well as study duration, since each may affect the efficacy of the treatment. It is also recommended to select a more clearly defined and narrow population for future studies. Another option to reveal the impact of these counfounding factors is to perform post-hoc analyses in studies. In addition, in amblyopia studies, there are practical barriers to randomization, blindness and obtaining complete and accurate data; it is also difficult to eliminate all forms of biases, specifically those caused by compliance. Thus, non-randomized studies with clearly defined inclusion criteria and clear protocol are also required in the future. On the basis of these sufficient and robust clinical trials, we can conduct more qualitative and quantitative reviews and provide high-level evidence for the treatment of amblyopia.

\section{Conclusions}

Differences in clinical efficacy among various amblyopia treatments might exist but could not be readily confirmed from the available data. According to the rankings, traditional patching regimens are probably more effective than atropine, unless atropine is used in combination with suppression of the sound eye. And the addition of activities appears to enhance the effect of patching. Further larger high-quality clinical trials are warranted in order to establish their efficacy with a higher degree of credibility.

\section{Supplementary information}

Supplementary information accompanies this paper at https://doi.org/10. 1186/s12886-020-01442-9.

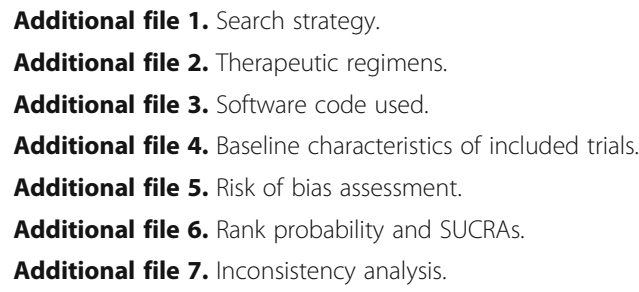

Additional file 1. Search strategy.

Additional file 2 . Therapeutic regimens

Additional file 3. Software code used.

Additional file 4. Baseline characteristics of included trials.

Additional file $\mathbf{5}$. Risk of bias assessment.

Additional file 6. Rank probability and SUCRAs.

Additional file 7. Inconsistency analysis.

\section{Abbreviations}

NMA: Network meta-analysis; RCT: Randomized controlled trial; BCVA: Best corrected visual acuity; MD: Mean difference; Cl: Confidence interval; Crl: Credibility interval; SD: Standard difference; H: Hours per day; Atr: Atropine; N: Near activities; D: Distant activities; Plano: Plano lens over the sound eye; log MAR: Logarithm of minimal angle of resolution; TCA: Traditional Chinese acunpture

\section{Acknowledgements}

Not applicable.

\section{Authors' contributions}

$L Y H, S H, Z X J$ and $L J$ were involved in the design, analysis and manuscript preparation. LYH, ZXJ and SYN were involved in the literature searching, screening and data collection. YTQ and WXY assessed the quality of included studies. ZXQ was involved in running the software. All authors were involved in the development of tmanuscript and have read and approved the final version.

\section{Funding}

The authors declare that they receive no funding from any corporation, companies or institutions etc.

\section{Availability of data and materials}

The datasets used and/or analyzed during the current study are available from the corresponding author on reasonable request.

Ethics approval and consent to participate

Not applicable.

Consent for publication

Not applicable.

\section{Competing interests}

The authors declare that they have no competing interests.

\section{Author details}

${ }^{1}$ Department of Evidence-Based Medicine and Clinical Epidemiology, West China Hospital, Sichuan University, No.37 Guoxue Alley, Chengdu 610041, China. ${ }^{2}$ Mental Health Center and Psychiatric Laboratory, the State Key Laboratory of Biotherapy, West China Hospital of Sichuan University, No.37 Guoxue Alley, Chengdu 610041, China. Department of Ophthalmology, West China Hospital, Sichuan University, No.37 Guoxue Alley, Chengdu 610041, China. ${ }^{4}$ Department of Clinical Research Management, West China Hospital, Sichuan University, No.37 Guoxue Alley, Chengdu 610041, China.

Received: 28 November 2019 Accepted: 22 April 2020

Published online: 25 May 2020

\section{References}

1. Holmes JM, Clarke MP. Amblyopia. Lancet. 2006;367(9519):1343-51.

2. Solebo AL, Cumberland PM, Rahi JS. Whole-population vision screening in children aged 4-5 years to detect amblyopia. Lancet. 2015;385(9984):2308-19.

3. Faghihi M, Hashemi H, Nabovati P, Saatchi M, Yekta A, Rafati S, et al. The prevalence of amblyopia and its determinants in a population-based study. Strabismus. 2017;25(4):176-83. 
4. Koo EB, Gilbert AL, VanderVeen DK. Treatment of amblyopia and amblyopia risk factors based on current evidence. Semin Ophthalmol. 2017;32(1):1-7.

5. Carlton J, Kaltenthaler E. Amblyopia and quality of life: a systematic review. Eye (Lond). 2011;25(4):403-13.

6. Birch EE, Castaneda YS, Cheng-Patel CS, Morale SE, Kelly KR, Beauchamp CL, et al. Self-perception of school-aged children with amblyopia and its association with reading speed and motor skills. JAMA Ophthalmol. 2019; 137(2):167-74.

7. Chen AM, Cotter SA. The amblyopia treatment studies: implications for clinical practice. Adv Ophthalmol Optom. 2016;1(1):287-305.

8. Foss AJE. Use of video games for the treatment of amblyopia. Curr Opin Ophthalmol. 2017;28(3):276-81.

9. Suttle CM. Active treatments for amblyopia: a review of the methods and evidence base. Clin Exp Optom. 2010;93(5):287-99.

10. Gaier ED, Hunter DG. Advances in amblyopia treatment: paradigm shifts and future directions. Int Ophthalmol Clin. 2017;57(4):117-28.

11. Hoyt C. What is next in amblyopia treatment? Ophthalmology. 2015;122(5):871-3.

12. Felius J, Chandler DL, Holmes JM, Chu RH, Cole SR, Hill M, et al. Evaluating the burden of amblyopia treatment from the parent and child's perspective. J AAPOS. 2010;14(5):389-95.

13. Hatt S, Antonio-Santos A, Powell C, Vedula SS. Interventions for stimulus deprivation amblyopia. Cochrane Database Syst Rev. 2006;3:CD005136.

14. Yazdani N, Sadeghi R, Momeni-Moghaddam H, Zarifmahmoudi L, Ehsaei A, Barrett BT. Part-time versus full-time occlusion therapy for treatment of amblyopia: a meta-analysis. J Curr Ophthalmol. 2017;29(2):76-84.

15. Li T, Shotton K. Conventional occlusion versus pharmacologic penalization for amblyopia. Cochrane Database Syst Rev. 2009;4:CD006460.

16. Liu L, Yang X, Luo D, Liao M, Chen B. Efficacy and tolerance of levodopa to treat amblyopia: a systematic review and meta-analysis. Eur J Ophthalmol. 2013;23(1):19-26.

17. Shotton K, Elliott S. Interventions for strabismic amblyopia. Cochrane Database Syst Rev. 2008;2:CD006461.

18. Yan X, Zhu T, Ma C, Liu A, Dong L, Wang J. A meta-analysis of randomized controlled trials on acupuncture for amblyopia. Evid Based Complement Alternat Med. 2013;2013:648054.

19. Hutton B, Salanti G, Caldwell DM, Chaimani A, Schmid CH, Cameron C, et al. The PRISMA extension statement for reporting of systematic reviews incorporating network meta-analyses of health care interventions: checklist and explanations. Ann Intern Med. 2015;162(11):777-84.

20. Beck RW, Moke PS, Turpin AH, Ferris FL, SanGiovanni JP, Johnson CA. A computerized method of visual acuity testing: adaptation of the early treatment of diabetic retinopathy study testing protocol. Am J Ophthalmol. 2003;135(2):203-5.

21. Holmes JM, Beck RW, Repka MX, Leske DA, Kraker RT, Blair RC, et al. The amblyopia treatment study visual acuity testing protocol. Arch Ophthalmol. 2001;119:1345-53.

22. Higgins JP, Altman DG, Gotzsche PC, Juni P, Moher D, Oxman AD, et al. The Cochrane Collaboration's tool for assessing risk of bias in randomised trials. BMJ. 2011;343:d5928.

23. Valkenhoef GV, Lu G, de Brock B, Hillege H, Ades AE, Welton NJ. Automating network meta-analysis. Res Synth Methods. 2012;3(4):285-99.

24. Dias S, Sutton AJ, Ades AE, Welton NJ. Evidence synthesis for decision making 2: a generalized linear modeling framework for pairwise and network meta-analysis of randomized controlled trials. Med Decis Mak. 2013;33(5):607-17

25. NJW SD, Caldwellb DM, Ades AE. Checking consistency in mixed treatment comparison meta-analysis. Stat Med. 2010;29:932-44.

26. Chaimani A, Mavridis D, Salanti G. A hands-on practical tutorial on performing meta-analysis with Stata. Evid Based Ment Health. 2014;17(4):111-6.

27. Glaser SR, Matazinski AM, Sclar DM, Sala NA, Vroman CM, Tanner CE, et al. A randomized trial of atropine vs patching for treatment of moderate amblyopia in children. Arch Ophthalmol. 2002;120(3):268-78.

28. Beck RW. A randomized trial of prescribed patching regimens for treatment of severe amblyopia in children. Ophthalmology. 2003; 110(11):2075-87.

29. Repka MX, Beck RW, Holmes JM, Birch EE, Chandler DL, Cotter SA, et al. A randomized trial of patching regimens for treatment of moderate amblyopia in children. Arch Ophthalmol. 2003;121(5):603-11.

30. Repka MX, Cotter SA, Beck RW, Kraker RT, Birch EE, Everett DF, et al. A randomized trial of atropine regimens for treatment of moderate amblyopia in children. Ophthalmology. 2004;111(11):2076-85.
31. Holmes JM, Edwards AR, Beck RW, Arnold RW, Johnson DA, Klimek DL, et al. A randomized pilot study of near activities versus non-near activities during patching therapy for amblyopia. J AAPOS. 2005;9(2):129-36.

32. Wallace DK, Edwards AR, Cotter SA, Beck RW, Arnold RW, Astle WF, et al. A randomized trial to evaluate 2 hours of daily patching for strabismic and anisometropic amblyopia in children. Ophthalmology. 2006;113(6):904-12.

33. Stewart CE, Stephens DA, Fielder AR, Moseley MJ, Cooperative R. Objectively monitored patching regimens for treatment of amblyopia: randomised trial. BMJ. 2007:335(7622):707.

34. Tejedor J, Rodriguez JM. Comparative efficacy of penalization modalities in moderate to mild amblyopia. Am J Ophthalmol. 2007;145(3):562-9.

35. Wallace DK, Lazar EL, Holmes JM, Repka MX, Cotter SA, Chen AM, et al. A randomized trial of increasing patching for amblyopia. Ophthalmology. 2013;120(11):2270-7.

36. Holmes JM, Manh VM, Lazar EL, Beck RW, Birch EE, Kraker RT, et al. Effect of a binocular iPad game vs part-time patching in children aged 5 to 12 years with amblyopia: a randomized clinical trial. JAMA Ophthalmol. 2016;134(12):1391-400.

37. Kelly KR, Jost RM, Dao L, Beauchamp CL, Leffler JN, Birch EE. Binocular Pad game vs patching for treatment of amblyopia in children: a randomized clinical trial. JAMA Ophthalmol. 2016;134(12):1402-8.

38. Gao TY, Guo CX, Babu RJ, Black JM, Bobier WR, Chakraborty A, et al. Effectiveness of a binocular video game vs placebo video game for improving visual functions in older children, teenagers, and adults with amblyopia: a randomized clinical trial. JAMA Ophthalmol. 2018;136(2):172-81.

39. Awan M, Proudlock FA, Gottlob I. A randomized controlled trial of unilateral strabismic and mixed amblyopia using occlusion dose monitors to record compliance. Invest Ophthalmol Vis Sci. 2005;46(4):1435-9.

40. Manh VM, Holmes JM, Lazar EL, Kraker RT, Wallace DK, Kulp MT, et al. A randomized trial of a binocular iPad game versus part-time patching in children aged 13 to 16 years with amblyopia. Am J Ophthalmol. 2018;186:104-15.

41. Menon V, Shailesh G, Sharma P, Saxena R. Clinical trial of patching versus atropine penalization for the treatment of anisometropic amblyopia in older children. J AAPOS. 2008;12(5):493-7.

42. Wallace DK, Lazar EL, Repka MX, Holmes JM, Kraker RT, Hoover DL, et al. A randomized trial of adding a Plano lens to atropine for amblyopia. J AAPOS. 2015;19(1):42-8

43. Rajavi Z, Sabbaghi H, Amini Sharifi E, Behradfar N, Yaseri M. The role of interactive binocular treatment system in amblyopia therapy. J Curr Ophthalmol. 2016;28(4):217-22

44. Herbison N, Mackeith D, Vivian A, Purdy J, Fakis A, Ash IM, et al. Randomised controlled trial of video clips and interactive games to improve vision in children with amblyopia using the I-BiT system. Br J Ophthalmol. 2016;100(11):1511-6.

45. Cotter SA, Weakley DR Jr, Strauber SF, Beck RW, Birch EE, Donahue S, et al. Pharmacological plus optical penalization treatment for amblyopia: results of a randomized trial. Arch Ophthalmol. 2009;127(1):22-30.

46. Scheiman MM, Hertle RW, Kraker RT, Beck RW, Birch EE, Felius J, et al. Patching Vs Atropine to Treat Amblyopia in Children Aged 7 to 12 Years. Arch Ophthalmol. 2008;126(12):1634-42.

47. Repka MX, Kraker RT, Beck RW, Birch E, Cotter SA, Holmes JM, et al. Treatment of severe amblyopia with weekend atropine: results from 2 randomized clinical trials. J AAPOS. 2009;13(3):258-63.

48. Pediatric Eye Disease Investigator Group. A randomized trial of near versus distance activities while patching for amblyopia in children aged 3 to less than 7 years. Ophthalmology. 2008;115(11):2071-8.

49. Holmes JM, Manny RE, Lazar EL, Birch EE, Kelly KR, Summers Al, et al. A randomized trial of binocular dig rush game treatment for amblyopia in children aged 7 to 12 years. Ophthalmology. 2019;126(3):456-66.

50. Beck RW. The pediatric eye disease Investigator Group. J AAPOS. 2000;2(5): 255-6.

51. Holmes JM. Designing clinical trials for amblyopia. Vis Res. 2015;114:41-7.

52. Li T, Qureshi R, Taylor K. Conventional occlusion versus pharmacologic penalization for amblyopia. Cochrane Database Syst Rev. 2019;8:CD006460.

53. Osborne DC, Greenhalgh KM, Evans MJE, Self JE. Atropine penalization versus occlusion therapies for unilateral amblyopia after the critical period of visual development: a systematic review. Ophthalmol Therapy. 2018;7(2): 323-32.

54. Shotton K, Powell C, Voros G, Hatt SR. Interventions for unilateral refractive amblyopia. Cochrane Database Syst Rev. 2008;4:CD005137.

55. Taylor K, Elliott S. Interventions for strabismic amblyopia. Cochrane Database Syst Rev. 2011;8:CD006461. 
56. Li SL, Jost RM, Morale SE, De La Cruz A, Dao L, Stager D Jr, et al. Binocular iPad treatment of amblyopia for lasting improvement of visual acuity. JAMA Ophthalmol. 2015;133(4):479-80.

57. Wang SP, Li QX, Li S. Systematic evaluation of levodopa effect on visual improvement in amblyopia: a meta-analysis. Clin Neuropharmacol. 2020; 43(1):20-25.

58. Cao ZX, Liu AG, Zhu TT, Li XJ, Wei YT, Yan XK. Molecular biology of the treatment of amblyopia with acupuncture: a review of recent research. Zhen Ci Yan Jiu. 2018;43(3):189-93.

\section{Publisher's Note}

Springer Nature remains neutral with regard to jurisdictional claims in published maps and institutional affiliations.

Ready to submit your research? Choose BMC and benefit from:

- fast, convenient online submission

- thorough peer review by experienced researchers in your field

- rapid publication on acceptance

- support for research data, including large and complex data types

- gold Open Access which fosters wider collaboration and increased citations

- maximum visibility for your research: over $100 \mathrm{M}$ website views per year

At $\mathrm{BMC}$, research is always in progress.

Learn more biomedcentral.com/submissions 